\title{
The Psychological Impact of Remote Communication on Body- Image Perception: Cosmetic Surgery on the Rise
}

\author{
Roxanne H. Padley ${ }^{1,2}$ ([) Bruno Di Pace ${ }^{3,4,5}$ (D)
}

\begin{abstract}
In recent times the number of requests for teleconsultations with plastic surgeons in private practice $(70 \%$ in the UK and $64 \%$ in the USA) has increased. The correlation between this increase and the concept of "Zoom Boom" changing how we perceive our image is under speculation. However, this could also be linked to the psychological impact of the pandemic on patients' moods. This study investigated the correlation between the pandemic era and seeking cosmetic surgery while focusing on the psychological impact of the pandemic on body-image perception. An anonymous web-based poll was distributed through social networks in Italy and the UK to patients seeking cosmetic surgery. The questions gathered responses on: 1. patient demographics; 2. cosmetic procedures requested; 3. motivations; 4. delays to cosmetic surgery caused by the pandemic; 5. pandemic effects on mood; 6 . influence of video conferencing on body-image perception. A total of 159 respondents completed the online poll.
\end{abstract}

Bruno Di Pace

dottbrunodipace@gmail.com

1 Department of Humanities, PhD School of Literary, Linguistic and Historical Studies, University of Salerno, Salerno, Italy

2 Linguistic Profiling for Professionals Research Unit, School of English, University of Nottingham, Nottingham, UK

3 Department of Medicine, Surgery and Dentistry "Scuola Medica Salernitana", PhD School of Translational Medicine of Development and Active Aging, University of Salerno, Via Giovanni Paolo II, 132, 84084 Fisciano, SA, Italy

4 Department of Plastic and Reconstructive Surgery, Addenbrooke's Hospital Cambridge University Hospitals NHS Foundation Trust, Cambridge, UK

5 Anglia Ruskin School of Medicine, Anglia Ruskin University, Cambridge \& Chelmsford, UK
Patients were more inclined to undergo cosmetic surgery during the pandemic citing the wish to improve their "lockdown face" (61\%) and also the benefits of home recovery during smart working $(36 \%)$. Eighty-one percent of respondents stated that video conferencing had impacted on their body-image perception, $95 \%$ indicated lower mood due to backlogs and surgery delays, while $72 \%$ of participants noted declined body-image perception during the pandemic. Data suggest that although video conferencing appears to play a key role in rising requests for cosmetic surgery consultations, a patient's negative self-perception is also likely linked to the psychological impact of the pandemic on mood levels.

Level of Evidence $V$ This journal requires that authors assign a level of evidence to each article. For a full description of these Evidence-Based Medicine ratings, please refer to the Table of Contents or the online Instructions to Authors www.springer.com/00266.

Keywords Teleconsultations · Cosmetic surgery - Zoom boom $\cdot$ Lockdown face $\cdot$ Psychological impact - Pandemic

The evolution in communication over the last 18 months has been transformational leading to adapting to the use of video conferencing platforms in almost all fields, perhaps of most note, education and medicine [1-3]. Indeed, this increase in remote video interaction has also led to an upturn in the number of requests for teleconsultations with plastic surgeons in private practice $(70 \%$ in the UK and $64 \%$ in the USA) [4-8]. There has been speculation surrounding the possible correlation between this increase in seeking cosmetic surgery being linked to the concept of "lockdown face" and whether constant exposure to one's 
image in video conferencing has led to changes in the way we perceive our own image, perhaps even distorting it $[7,9]$. This potential change in our self-perception, however, could be linked not only to the time spent looking at our own image and noticing one's flaws but also the psychological impact of the pandemic on patients' moods $[7,10]$.

Previous literature within the field of breast cancer and reconstruction has identified a clear correlation between mood levels and women's body-image perception [10]. Moreover, the decision by the majority of healthcare systems to defer elective surgery during the pandemic, including breast reconstruction [11-13], has also likely worsened this psychological impact. Although a direct link between the psychological well-being of patients seeking cosmetic surgery as a result of the "Zoom Boom" and patients undergoing mastectomies and reconstructions [14-21] may not be immediate, particularly as oncoplastic patients face greater psychological challenges, self-isolation and lower moods during the pandemic should not be ruled out as potential motives for expressing a desire to enhance one's appearance. Therefore, this study investigated the correlation between the pandemic era and seeking cosmetic surgery with a specific focus on the psychological impact of the pandemic on body-image perception.

An anonymous web-based poll was designed and distributed through social networks in both Italy and the UK throughout the month of May 2021. The questions were designed to gather responses on the following areas: 1 . patient demographics (age, gender, location, highest qualification); 2. cosmetic procedure being sought; 3 . reasons for seeking cosmetic surgery; 4 . delays to cosmetic surgery caused by the SARS-COV-2 pandemic; 5. pandemic effects on mood; 6 . the influence of video conferencing on body-image perception.

A total of 159 respondents completed the online poll ( $73 \%$ females and $27 \%$ males), and the mean age of participants was 43 years old; $56 \%$ were based in the UK and $44 \%$ in Italy; $57 \%$ of respondents had an undergraduate degree (minimum three years of study). The types of surgeries indicated were predominantly to the upper body and face $(78 \%)$ with almost two-thirds of these opting for minor facial procedures $(56 \%$ botulinum toxin injection; $32 \%$ filler injections; $12 \%$ both), and the other-third were evenly orientated toward breast surgery and other more invasive facial procedures (rhinoplasty and rhytidectomy). Other desirable procedures included lower body interventions (liposuction at $15 \%$ and abdominoplasty $7 \%$ ).

Patients were more inclined to undergo cosmetic surgery during the pandemic mainly due to both their wish to improve their "lockdown face" for video conferencing $(61 \%)$ and also due to the benefits of home recovery while in smart working (36\%). Furthermore, although $81 \%$ of respondents stated that video conferencing had impacted on their body-image perception, delays and access to surgeons during the pandemic were perceived as having a negative impact on patient's moods (95\%) and participants claimed that their body-image perception and self-esteem were lower than in the pre-pandemic, particularly during lockdown $(72 \%)$.

The data indicate that the majority of patients state they are seeking cosmetic surgery in order to improve their "lockdown face" and that video conferencing has indeed negatively influenced their body-image perception [7]. This is also supported by the figures regarding the type of surgery requested as $69 \%$ of patients indicated that they are seeking a facial procedure (either minor or major). This would therefore confirm the hypothesis that the influence of video conferencing has led to an increase in requests for consultations with plastic surgeons (even if these do not necessarily materialize into surgeries).

However, the cause for such an increase cannot purely be attributed to time spent on online meetings noticing flaws, but other factors should be evaluated. Indeed, the $31 \%$ of respondents who were seeking non-facial surgery were likely not influenced by video conferencing and it is pertinent to consider that mood levels were also one of the motivating factors. This is confirmed by the fact that almost all patients (95\%) indicate that delays, backlogs and access to surgeons during the pandemic impacted negatively on their mood and $72 \%$ of patients confirmed that the pandemic caused both lower self-esteem and worsened perception of their body-image in general (not just facially).

Therefore, even though video conferencing would appear to play a key role in the increase in requests for cosmetic surgery consultations, our results suggest that a patient's negative self-perception is also likely linked to the psychological impact of the pandemic on moods and potential levels of depression. It is thus paramount that plastic surgeons carry out a correct evaluation of the actual motivating factor which leads a patient to request cosmetic surgery and their overall well-being in order to fully meet patient expectations and avoid discontentment with the aesthetic outcomes. It would be advisable for further studies to be advanced in this sense in order to continue to guarantee the high ethical standards and safety of patients who undergo cosmetic surgery. 
Author Contributions RHP and BDP jointly contributed to the literature review, manuscript preparation, editing and approval.

\section{Declarations}

Conflict of interest The authors declare that they have no conflict of interest.

Human or Animal Rights This article does not contain any studies with human participants or animals performed by any of the authors.

Informed Consent For this type of study, informed consent is not required.

\section{References}

1. Padley RH, Di Pace B (2021) COVID-19 lockdown learning: the revolution of virtual teaching. J Plast Reconstr Aesthet Surg 74(2):407-447

2. Padley RH, Di Pace B (2021) Four strategies for plastic surgery education amid the COVID-19 pandemic. Plast Reconstr Surg 148(1):152e-153e

3. Padley RH, Di Pace B (2021) Medical advances in telehealth and innovative communication: new directions. Gazz Med Ital Arch Sci Med 180(4):99-101

4. Gillman-Wells CC, Sankar TK, Vadodaria S (2021) COVID-19 Reducing the risks: telemedicine is the new norm for surgical consultations and communications. Aesthet Plast Surg 45(1):343-348

5. British Association of Aesthetic Plastic Surgeons. "Zoom Boom" - UK cosmetic surgeons see massive rise in demand for online consultations but urge caution. Available at: https:// pressreleases.responsesource.com/news/99857/zoom-boom-uk-cos metic-surgeons-see-massive-rise-in-demand/

6. American Society of Plastic Surgeons. ASPS Predicts New Industry Trends Amidst COVID-19 Reopenings. Available at: https://www.plasticsurgery.org/news/press-releases/american-soc iety-of-plastic-surgeons-predicts-new-industry-trends-amidst-covid 19-reopenings

7. Padley RH, Di Pace B (2021) Touch-ups, rejuvenation, re-dos and revisions: remote communication and cosmetic surgery on the rise. Aesthet Plast Surg. https://doi.org/10.1007/s00266-02102235-1

8. Verdura V, Di Pace B, Cagiano L, Portincasa A (2021) Home wound management during the COVID-19 pandemic: the use of a novel adhesive retention suture. JAAD Case Rep 9:67-68

9. Imam SZ, Karanasios G, Khatib M, Cavale N, Amar O, Mayou B (2021) Resumption of cosmetic surgery during covid- experience of a specialised cosmetic surgery day-case hospital. J Plast Reconstr Aesthet Surg. https://doi.org/10.1016/j.bjps. 2021.03.070

10. Di Pace B, Marotta M, Serlenga G et al (2020) Breast cancer and reconstruction: it changes inside and out. J Plast Reconstr Aesthet Surg 73(2):391-407

11. Di Pace B, Benson JR, Malata CM (2020) Breast reconstruction and the COVID-19 pandemic: a viewpoint. J Plast Reconstr Aesthet Surg 73(7):1357-1404

12. Di Pace B, Benson JR, Malata CM (2021) Breast reconstruction and the COVID-19 pandemic: adapting practice. J Plast Reconstr Aesthet Surg 74(3):644-710

13. Rocco N, Montagna G, Di Micco R et al (2021) The impact of the COVID-19 pandemic on surgical management of breast cancer: Global trends and future perspectives. Oncologist 26(1):e66-e77

14. Rubino C, Faenza M, Di Pace B, Campitiello N, Brongo S, Zingone G (2017) A new keystone flap "Plus" design: case series and analysis of follow-up. J Plast Reconstr Aesthet Surg 70(7):976-979

15. Di Pace B, Rubino C (2021) The modified keystone flap in periarticular or large superficial tumor resection surgery. J Surg Oncol 123(4):1164-1165

16. Di Pace B, Verdura V, Concilio M et al (2017) A rare case of squamous cell carcinoma of a proliferating trichilemmal tumour. Ann Ital Chir 88:263-267

17. Verdura V, Di Pace B, Concilio M et al (2019) A new case of radiation-induced breast angiosarcoma. Int J Surg Case Rep 60:152-155

18. Ngaage LM, Oni G, Di Pace B et al (2018) The effect of CT angiography and venous couplers on surgery duration in microvascular breast reconstruction: a single operator's experience. Gland Surg 7(5):440-448

19. Ngaage LM, Hamed R, Oni G et al (2019) The role of CT angiography in assessing deep inferior epigastric perforator flap patency in patients with pre-existing abdominal scars. J Surg Res 235:58-65

20. Guastafierro A, Verdura V, Di Pace B, Faenza M, Rubino C (2019) The influence of breast cancer on the distribution of cherry angiomas on the anterior thoracic wall: a case series study. Dermatology 235(1):65-70

21. Di Pace B, Khan F, Patel M et al (2021) A multicentre study of the relationship between abdominal flap and mastectomy weights in immediate unilateral free flap breast reconstruction and the effect of adjuvant radiotherapy. J Plast Reconstr Aesthet Surg. https://doi.org/10.1016/j.bjps.2021.06.010

Publisher's Note Springer Nature remains neutral with regard to jurisdictional claims in published maps and institutional affiliations. 